\title{
Incremental Healthcare Utilization and Cost Burden of Comorbid Insomnia in Alzheimer's Disease Patients
}

\author{
Zaina P. Qureshi ${ }^{\mathrm{a}, *}$, Ellen Thiel ${ }^{\mathrm{b}}$, James Nelson ${ }^{\mathrm{b}}$ and Rezaul Khandker ${ }^{\mathrm{a}}$ \\ ${ }^{a}$ Merck \& Co., Inc., Kenilworth, NJ, USA \\ ${ }^{\mathrm{b}}$ IBM Watson Health, Cambridge, MA, USA
}

Accepted 23 July 2021

Pre-press 20 August 2021

\begin{abstract}
.
Background: Insomnia is associated with worsened clinical outcomes among Alzheimer's disease dementia (AD) patients, increased caregiver burden, and healthcare utilization.

Objective: This study aimed to characterize the incremental healthcare burden of insomnia in AD using real-world data.

Methods: A retrospective observational study was conducted on AD patients selected from the IBM ${ }^{\circledR}$ MarketScan Commercial and Medicare Supplemental Databases. AD patients with claims-based evidence of insomnia were direct matched to a non-insomnia cohort based on demographic factors. Healthcare utilization and associated costs were assessed for a 12-month follow-up period.

Results: A total of 3,500 insomnia AD patients and 9,884 non-insomnia AD patients were analyzed. The insomnia cohort had a higher comorbidity burden at baseline (mean score on Charlson Comorbidity Index 2.5 versus $2.2, p<0.001$ ) and higher proportions of patients with baseline diagnoses for other conditions including depression: $40 \%$, insomnia cohort versus $25 \%$, non-insomnia $(p<0.001)$. AD patients with insomnia were more likely to have a claim for inpatient hospitalizations $(39.8 \%$ versus $32.3 \%$ ), emergency room services $(56.4 \%$ versus $48.0 \%)$, and skilled-nursing services $(42.6 \%$ versus $31.9 \%$ ) (all $p<0.05)$. Mean total annual healthcare costs during the 12-month follow-up period were significantly higher among AD patients with insomnia as compared to those without. (Mean costs: $\$ 37,356$ versus $\$ 27,990, p<0.001$ ).

Conclusion: AD patients with comorbid insomnia are more likely to use higher-cost healthcare services such as inpatient hospitalization, and skilled nursing, and have higher total healthcare costs. This real-world analysis provides evidence that $\mathrm{AD}$ disease management should consider proper treatment of comorbid insomnia due to the incremental burden and cost implications.
\end{abstract}

Keywords: Alzheimer's disease, dementia, facilities and services utilization, health care economics, sleep initiation and maintenance disorders

\section{INTRODUCTION}

Alzheimer's disease (AD) is a neurodegenerative disease and the leading cause of dementia in the elderly [1,2]. The Alzheimer's Association estimated there were 5.8 million Americans age 65 and older living with $\mathrm{AD}$ in 2019 [3], $81 \%$ of which were at

\footnotetext{
${ }^{*}$ Correspondence to: Zaina P. Qureshi, Merck \& Co., Inc., 2000 Galloping Hill Rd, Kenilworth, NJ 07033, USA. Tel.: +1 908873 9567; E-mail: zaina.qureshi@ merck.com.
}

least 75 years old [4]. The population of people over 65 in the United States living with AD is projected to grow to $12.7-13.8$ million by $2050[4,5]$.

The classic hallmarks of $\mathrm{AD}$ are progressive deterioration of memory, intellect, and motor skills leading to increasing disability and dependency on caregivers $[3,6]$. Patients with AD have increased use of healthcare services, including more frequent highcost events like hospitalizations and emergency room visits, compared to cognitively normal individuals [7]. This increased need for care creates a significant 
financial burden. In 2019 the costs associated with healthcare and long-term care for individuals with dementia due to $\mathrm{AD}$ and other causes were estimated to be $\$ 290$ billion, with the average Medicare beneficiary with AD costing $\$ 35,000$ more annually than Medicare beneficiaries without AD [3].

Sleep disorders such as insomnia occur more frequently in $\mathrm{AD}$ patients than in the general population [8]. Between $25 \%-40 \%$ of patients with AD report having some type of sleep disorder, such as excessive awakenings, early morning awakenings, excessive daytime sleepiness, and napping for more than $1 \mathrm{~h}$ during the day $[9,10]$. A recent meta-analysis found that $20 \%$ of patients with dementia living in hospice care had clinically significant sleep disturbances, $38 \%$ had any symptom of sleep disturbance, and 70\% had poor sleep efficiency as measured by actigraphy [11].

The relationship between sleep and AD is theorized to be bidirectional, with poor sleep as both a cause and consequence of AD [12]. Sleep disturbances often appear early in the course of $\mathrm{AD}$ progression and tend to be correlated with the rate of cognitive decline [13]. Conversely, poor sleep in older adults may result in cognitive decline that could progress to $\mathrm{AD}[14,15]$.

In patients with $\mathrm{AD}$, sleep disturbances are associated with worsening behavioral and psychological symptoms, which may lead to increased caregiver burden, increased fall risk, and increased use of highcost interventions such as skilled nursing support and early institutionalization [16, 17]. However, no studies have looked at the healthcare resource utilization and cost implications of insomnia in AD. In this study, we characterized the incremental healthcare burden of insomnia in $\mathrm{AD}$ using real-world data by comparing all-cause healthcare utilization and costs between a cohort of AD patients with insomnia to a matched cohort of AD patients without insomnia. Multivariable analysis was used to estimate all-cause total healthcare costs, odds of an inpatient admission, and odds of having a claim for skilled nursing facility services after adjusting for baseline differences between $\mathrm{AD}$ patients with and without insomnia.

\section{METHODS}

\section{Study design and data source}

This retrospective cohort study utilized de-identified US administrative claims data from the IBM MarketScan Commercial Claims and Encounters database (Commercial) and MarketScan Medicare Supplemental and Coordination of Benefits database (Medicare) for the period from January 1, 2014 to December 31, 2018. The Commercial database contains the inpatient, outpatient, and outpatient prescription drug experience of employees and their dependents, covered under a variety of fee-forservice and managed care health plans. The Medicare database contains the same healthcare experience of retirees with Medicare supplemental insurance paid for by employers. Both the Medicare-covered portion of payment (represented as Coordination of Benefits Amount) and the employer-paid portion are included in this database.

All database records are statistically de-identified and certified to be fully compliant with US patient confidentiality requirements set forth in the Health Insurance Portability and Accountability Act of 1996. Because this study used only de-identified patient records and did not involve the collection, use, or transmittal of individually identifiable data, this study was exempted from Institutional Review Board approval.

Relevant diagnoses, procedures, and treatments were obtained using International Classification of Diseases, 9th and 10th Revision, Clinical Modification (ICD-9-CM and ICD-10-CM) codes, Current Procedural Terminology 4th edition codes, Healthcare Common Procedure Coding System codes, and National Drug Codes.

\section{Patient selection and cohort assignment}

We identified patients with at least one inpatient or two outpatient claims with a diagnosis of AD (ICD9-CM: 331.0; ICD-10-CM: G30.0, G30.1, G30.8, G30.9) between January 1, 2015 and December 31, 2018 in the MarketScan databases. The date of the first claim with a diagnosis of AD was defined as the $\mathrm{AD}$ diagnosis date. To be eligible for study inclusion, we required that patients were 50-100 years old on the AD diagnosis date, had continuous enrollment with medical and pharmacy benefits for at least 12 months before and after the AD diagnosis date, and had no claims with a diagnosis of $\mathrm{AD}$ in the 12 months before the AD diagnosis date.

From this population of patients newly-diagnosed with $\mathrm{AD}$, we identified two populations: those with a diagnosis of insomnia or claim for an insomnia medication in the three months preceding or 12 months following the AD diagnosis date (insomnia cohort) and those without a diagnosis of insomnia 
or claim for an insomnia medication in the same period (non-insomnia cohort). The diagnosis codes for insomnia used in this analysis included ICD9-CM codes 327.00, 327.01, 327.02, 780.51, and 780.52 along with ICD-10-CM codes F51.01, F51.02, F51.03, F51.04, F51.05, F51.09, F51.8, F51.9, G47.00, G47.01, and G47.09. Prescription insomnia medications included benzodiazepines (estazolam, flurazepam, quazepam, temazepam, and triazolam) and non-benzodiazepines (low-dose doxepin [3-6 mg], eszopiclone, ramelteon, suvorexant, zaleplon, and zolpidem tartrate). The index date for the insomnia cohort was the date of the first claim with an insomnia diagnosis for all patients with an insomnia diagnosis or the date of the first claims for an insomnia medication for patients without an insomnia diagnosis but with a claim for prescription insomnia medication. The index date of controls was assigned after matching and set as the date corresponding to the same number of days between the matched insomnia patient's AD diagnosis date and insomnia diagnosis date.

We required that patients have continuous enrollment with medical and pharmacy benefits for at least 12 months before and after the index date, and we excluded patients with a diagnosis of a non-insomnia sleep disorder in the three months preceding or 12 months following the $\mathrm{AD}$ diagnosis date. Where possible, insomnia patients were directly matched (1:3) to non-insomnia patients on age, sex, region, and year of the index insomnia diagnosis. Due to sample limitations, it was not possible to identify three non-insomnia matched controls with sufficient continuous enrollment for every insomnia patient. Insomnia patients with no matches were excluded, while those with 1 or 2 matches were retained in the sample set.

\section{Outcomes}

Demographic characteristics were measured on the index date and included age and sex. Baseline clinical characteristics were measured during the 12-months preceding the index date and included Deyo-Charlson Comorbidity Index (DCI) and the presence of selected comorbid conditions representing common cost drivers and common neurological and psychological conditions, including cerebrovascular disease, congestive heart failure, depression, epilepsy, hyperlipidemia, hypertension, malignancy, mild liver disease, moderate or severe liver disease, mood disorder, myocardial infarction, other sleep disorders, Parkinson's disease, peripheral vascular disease, pneumonia, renal disease, sleep apnea, and substance abuse disorders. All-cause healthcare resource utilization, including inpatient admissions, outpatient services (emergency department [ED] visits, physician office visits, skilled nursing facility services, hospice services, and other outpatient services), and outpatient pharmacy services were recorded in the 12 months following the index date. All-cause costs for these claims were calculated from the paid amounts of fully adjudicated claims, including insurer and health plan payments, as well as patient cost-sharing in the form of copayment, deductible, and coinsurance. All costs are reported as per person per month (PPPM) and were inflated to 2018 US dollars using the medical care component of the Consumer Price Index [18].

\section{Statistical analysis}

Mean and standard deviation (SD) were reported for continuous variables, and statistical significance was determined using Student's $t$-tests. Frequencies and percentages were reported for categorical variables, and statistical significance was determined using Chi-square tests. The alpha level for all statistical tests was 0.05. All data analyses were conducted using WPS version 4.2 (World Programming, United Kingdom).

Multivariable analysis was used to examine primary healthcare utilization and cost outcomes while controlling for baseline demographic and clinical characteristics. The outcomes analyzed in separate generalized linear models included total all-cause healthcare costs, odds of an inpatient admission, and costs of a claim for skilled nursing facility services. Logistic regression was used to model the odds of an inpatient admission or skilling nursing facility claim occurring in the follow-up period. Costs were analyzed using a generalized linear model (GLM) with a log link function and a gamma error distribution. The main explanatory variable of interest was the presence of insomnia diagnosis. Each model adjusted for age, sex, and all baseline comorbid conditions listed in the outcomes section except epilepsy (due to rarity and similarity across cohorts), malignancy and renal disease (both due to extreme rarity in the cohorts).

To estimate the costs from the model in terms of dollars, two approaches were taken. The first was to use the dollar costs predicted by the model. The second was to use a counterfactual based approach where two copies of the full data set were made, with one 
being labelled the AD control group and the other the insomnia group. For the AD control copy the cost for those people who were in the insomnia group was changed to a counterfactual estimate of AD control cost based on the GLM coefficient only; and vice versa with the insomnia copy, i.e., the cost for the AD control people was changed to the counterfactual estimate for the insomnia group. Summary statistics for each of these copies were then used for estimates of the dollar costs for each person if they had had been in the other group. In most cases the counterfactual estimates of the group means were only modestly different from those observed or estimated from the model predictions.

\section{RESULTS}

There were 116,541 individuals in the MarketScan databases with a qualifying diagnosis of AD between January 1, 2015 and December 31, 2018 (Fig. 1). Of these, 112,466 were between the ages of 50 and 99 at their first AD diagnosis during the selection window. We excluded 61,128 individuals because they did not meet the initial continuous enrollment criteria and an additional 14,473 individuals because they had a prior diagnosis of AD. This left 36,865 qualifying adults with $\mathrm{AD}$, of which 5,374 (14.6\%) had a diagnosis of insomnia or claim for an insomnia medication in the 3 months preceding or 12 months following their AD diagnosis.

After excluding patients with evidence of other sleep disorders and those who did not have 12 months of continuous enrollment in MarketScan before and after their qualifying insomnia diagnosis or assigned proxy diagnosis date, there were 3,602 AD patients with insomnia and 25,312 AD patients without insomnia. The patients with insomnia were then directly matched on age, sex, region, and year of diagnosis to up to three patients without insomnia. AD patients with insomnia not directly matched to at least one non-insomnia patient were excluded from further analysis. This approach was used to balance patient characteristics and to maintain sample size of the insomnia cohort. A total of 3,500 AD patients with insomnia met all inclusion criteria and were matched to $9,884 \mathrm{AD}$ patients without insomnia (Fig. 1). This reflects the loss of $102 \mathrm{AD}$ patients due to lack of at least one direct match in the initial sample and an effective matching ratio of 1:2.82 among the remaining patients; Supplementary Table 1 shows the demographic characteristics of the pre-match group.
The mean age of AD patients in both the insomnia and non-insomnia cohorts was approximately 82 years, and about $67 \%$ of patients in both cohorts were female (Table 1). Patients with insomnia had a higher CCI in the baseline period compared to non-insomnia patients $(2.50$ versus $2.16, p<0.001)$ and were more likely to have a prior diagnosis of many comorbid conditions of interest, most notably depression $(39.5 \%$ versus $24.9 \%, p<0.001)$, moderate to severe liver disease $(36.8 \%$ versus $31.9 \%$, $p<0.001)$, and hypertension $(78.9 \%$ versus $74.3 \%$, $p<0.001)$. Patients with insomnia were also more likely to have a prior diagnosis of hyperlipidemia ( $53.7 \%$ versus $50.9 \%, p=0.004)$, peripheral vascular disease $(25.7 \%$ versus $22.6 \%, p<0.001)$, myocardial infarction $(18.2 \%$ versus $15.5 \%, p<0.001)$, mild liver disease ( $15.6 \%$ versus $12.2 \%, p<0.001)$, congestive heart failure $(13.9 \%$ versus $11.2 \%, p<0.001)$, pneumonia $(12.3 \%$ versus $8.5 \%, p<0.001)$, Parkinson's disease $(7.0 \%$ versus $5.2 \%, p<0.001)$, substance abuse disorders $(4.0 \%$ versus $1.9 \%, p<0.001)$, mood disorders $(4.0 \%$ versus $1.9 \%, p<0.001)$, other sleep disorders $(3.8 \%$ versus $2.1 \%, p<0.001)$, or cerebrovascular disease $(3.8 \%$ versus $2.9 \%, p=0.005)$.

AD patients with insomnia had higher all-cause healthcare utilization than $\mathrm{AD}$ patients without insomnia (Table 2). Patients with insomnia were more likely to have at least one inpatient admission $(39.8 \%$ versus $32.3 \%, p<0.001)$, had more frequent inpatient admissions (mean [SD]: 0.6 [0.9] versus 0.4 [0.7], $p<0.001$ ), and more days spent in the inpatient setting (9.1[13.5] versus 7.6[11.0], $p<0.001$ ). Patients with insomnia were also more likely to have greater utilization of ED services $(56.4 \%$ versus $48.0 \%, p<0.001)$, skilled nursing facilities $(42.6 \%$ versus $31.9 \%, p<0.001$ ), or other outpatient services $(98.5 \%$ versus $97 \%, p<0.001)$. While the percentage of patients with at least one outpatient office visit or hospice service claim was not significantly different between patients with or without insomnia, patients with insomnia had, on average, more office visits (mean [SD]: 8.8 [9.1] versus 7.5 [7.6], $p<0.001)$ and more hospice claims $(0.3$ [6.6] versus 0.1 [2.7], $p=0.012$ ) than patients without insomnia. In addition, patients with insomnia were more likely to have at least one outpatient pharmacy claim $(97.4 \%$ versus $95.3 \%, p<0.001)$ and had, on average, more outpatient pharmacy claims (65.3 claims versus 47.8 claims, $p<0.001)$.

AD patients with insomnia had higher mean (SD) and median unadjusted all-cause healthcare costs PPPY than AD patients without insomnia 
Patients $\geq 1$ inpatient claim or $\geq 2$ outpatient claims with a diagnosis of Alzheimer's disease (AD) ${ }^{1}$ between January 1, 2015 and December 31,2018 . The date of the first claim will be the $A D$ diagnosis date.

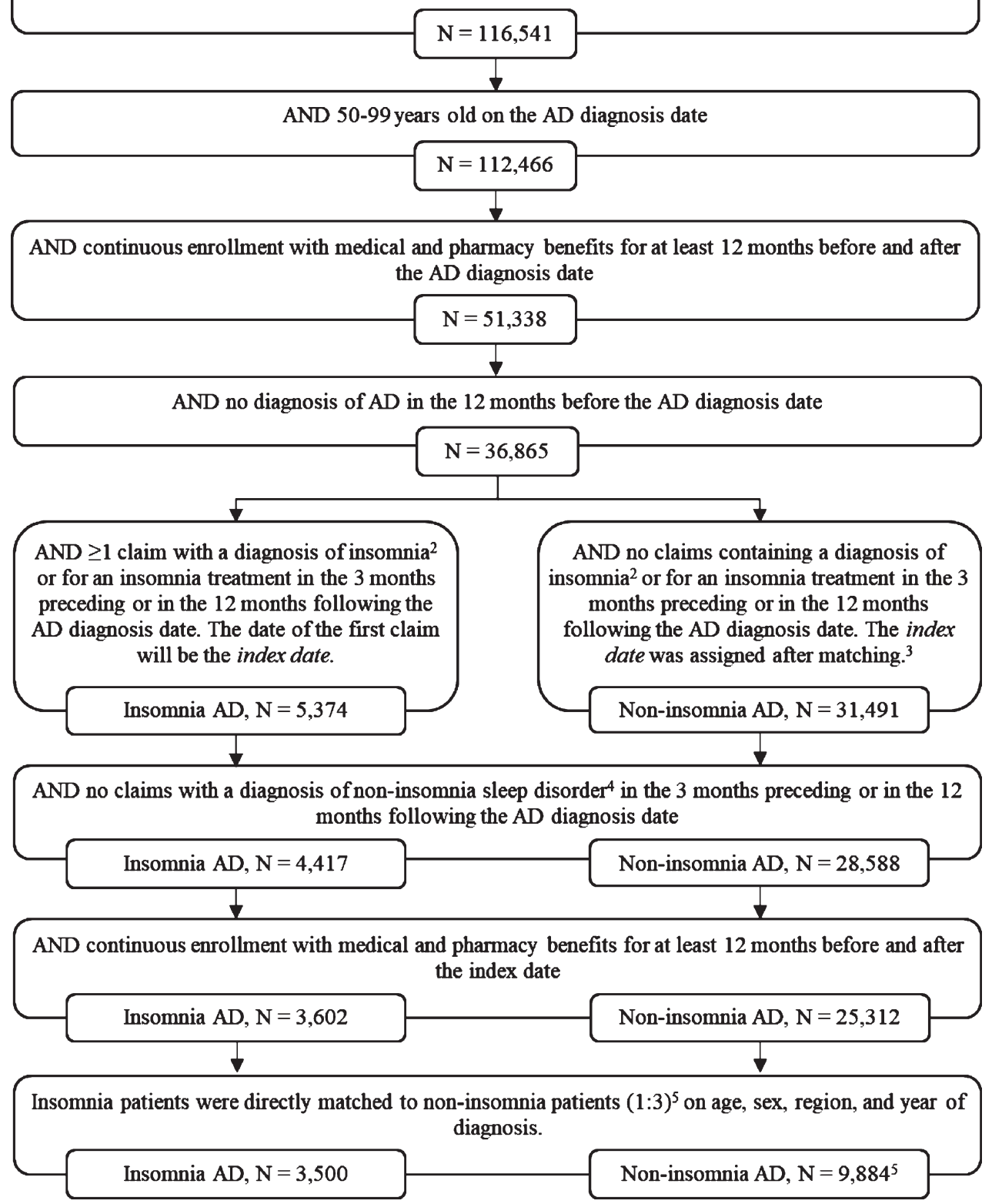

Fig. 1. Patient selection. ${ }^{1}$ Alzheimer's disease diagnosis codes: ICD-9-CM code 331.0; ICD-10-CM codes: G30.0, G30.1, G30.8, G30.9. ${ }^{2}$ Insomnia diagnosis codes: ICD-9-CM code 331.0; ICD-10-CM codes: G30.0, G30.1, G30.8, G30.9. ${ }^{3}$ Control patients will be assigned an index date corresponding to the same number of days between the matched insomnia patient's AD diagnosis and first treatment for insomnia (e.g., -3 months to +12 months). ${ }^{4}$ Non-insomnia sleep disorders include sleep apnea, narcolepsy, and others. ${ }^{5}$ Due to sample limitations it was not possible to identify three non-insomnia matched controls with sufficient continuous enrollment for every insomnia patient. Insomnia patients with no matches were excluded while those with 1 or 2 matches were retained in the sample set.

(mean [SD]: $\$ 37,356[\$ 70,089]$ versus $\$ 27,990$ [\$57,179], $p<0.001)$; median costs: $\$ 18,813$ versus \$12,318) (Fig. 2 and Table 3). The largest driver of higher costs among patients with insomnia were inpatient claims $(\$ 13,817[\$ 48,854]$ versus $\$ 9,657$
$[\$ 32,563], p<0.001)$. Costs were also higher for outpatient services overall $(\$ 18,546[\$ 36,351]$ versus $\$ 14,323[\$ 4,432], p<0.001)$, as well as all specific sub-categories of outpatient care except hospice: emergency room services $(\$ 2,514[\$ 8,932]$ versus 
Table 1

Demographics and baseline clinical characteristics

\begin{tabular}{|c|c|c|c|c|c|}
\hline \multirow[b]{3}{*}{ Age (Mean, SD) } & \multicolumn{2}{|c|}{ Insomnia patients } & \multicolumn{2}{|c|}{ Non-insomnia patients } & \multirow[t]{2}{*}{$p$} \\
\hline & \multicolumn{2}{|c|}{$N=3,500$} & \multicolumn{2}{|c|}{$N=9,884$} & \\
\hline & 81.7 & 8.6 & 82.0 & 8.2 & 0.077 \\
\hline Female & 2,334 & $66.7 \%$ & 6,651 & $67.3 \%$ & 0.513 \\
\hline \multicolumn{6}{|l|}{$\begin{array}{l}\text { Calendar year of } \mathrm{AD} \\
\text { diagnosis date }(N, \%)\end{array}$} \\
\hline 2015 & 1,777 & $50.8 \%$ & 5,057 & $51.2 \%$ & \multirow[t]{4}{*}{0.928} \\
\hline 2016 & 1,223 & $34.9 \%$ & 3,454 & $34.9 \%$ & \\
\hline 2017 & 499 & $14.3 \%$ & 1,369 & $13.9 \%$ & \\
\hline 2018 & 1 & $0.0 \%$ & 4 & $0.0 \%$ & \\
\hline $\begin{array}{l}\text { Charlson Comorbidity } \\
\text { Index (Mean, SD) }\end{array}$ & 2.50 & 2.23 & 2.16 & 2.10 & $<0.001$ \\
\hline \multicolumn{6}{|l|}{ Comorbid conditions $(N, \%)$} \\
\hline Hypertension & 2,761 & $78.9 \%$ & 7,345 & $74.3 \%$ & $<0.001$ \\
\hline Hyperlipidemia & 1,881 & $53.7 \%$ & 5,035 & $50.9 \%$ & 0.004 \\
\hline Depression & 1,384 & $39.5 \%$ & 2,460 & $24.9 \%$ & $<0.001$ \\
\hline Moderate or severe liver disease & 1,287 & $36.8 \%$ & 3,150 & $31.9 \%$ & $<0.001$ \\
\hline Peripheral vascular disease & 899 & $25.7 \%$ & 2,233 & $22.6 \%$ & $<0.001$ \\
\hline Myocardial infarction & 636 & $18.2 \%$ & 1,536 & $15.5 \%$ & $<0.001$ \\
\hline Mild liver disease & 546 & $15.6 \%$ & 1,208 & $12.2 \%$ & $<0.001$ \\
\hline Congestive heart failure & 488 & $13.9 \%$ & 1,106 & $11.2 \%$ & $<0.001$ \\
\hline Pneumonia & 430 & $12.3 \%$ & 844 & $8.5 \%$ & $<0.001$ \\
\hline Parkinson's disease & 244 & $7.0 \%$ & 514 & $5.2 \%$ & $<0.001$ \\
\hline Substance abuse disorders & 141 & $4.0 \%$ & 192 & $1.9 \%$ & $<0.001$ \\
\hline Mood disorder & 140 & $4.0 \%$ & 187 & $1.9 \%$ & $<0.001$ \\
\hline Other sleep disorders ${ }^{1}$ & 134 & $3.8 \%$ & 208 & $2.1 \%$ & $<0.001$ \\
\hline Cerebrovascular disease & 134 & $3.8 \%$ & 283 & $2.9 \%$ & 0.005 \\
\hline Epilepsy & 132 & $3.8 \%$ & 369 & $3.7 \%$ & 0.919 \\
\hline Sleep apnea ${ }^{1}$ & 61 & $1.7 \%$ & 135 & $1.4 \%$ & 0.111 \\
\hline Any malignancy & 27 & $0.8 \%$ & 60 & $0.6 \%$ & 0.298 \\
\hline Renal disease & 12 & $0.3 \%$ & 42 & $0.4 \%$ & 0.510 \\
\hline
\end{tabular}

Demographic information captured on index date. Clinical characteristics were measured during the 12-month pre-index baseline period.

${ }^{1}$ This captures the subset of patients with a diagnosis of other sleep disorders or sleep apnea during the variable period between the start of the 12-month pre-index period and the start of the 3-month pre-AD diagnosis exclusion period.

$\$ 1,730$ [\$6,334], $p<0.001$ ), outpatient office visits $(\$ 988$ [\$1,151] versus $\$ 839$ [\$1,007], $p<0.001)$, and skilled nursing facility claims $(\$ 5,333$ versus $\$ 3,674$, $p<0.001)$. The full cost results, including medians, can be found in Table 3 .

After adjusting for baseline differences in base line demographic and clinical characteristics, patients with insomnia had significantly higher odds $(95 \% \mathrm{CI})$ of an inpatient admission (1.38 [1.22-1.44], $p<$ 0.001 ) and of a skilled nursing facility claim (1.44 [1.32-1.56], $p<0.001)$ than patients without insomnia (Fig. 3). Similarly, patients with insomnia experienced $28 \%$ higher all-cause healthcare costs than patients without insomnia (1.28 [1.23-1.34], $p<0.001)$. Mean $(95 \% \mathrm{CI})$ model-adjusted predicted costs were $\$ 37,849(\$ 37,514-\$ 38,183)$ for patients with insomnia and $\$ 27,810(\$ 27,670-\$ 27,949)$ for patients without insomnia. The full results of the multivariable models can be found in Supplementary Table 2.

\section{DISCUSSION}

While the research literature on $\mathrm{AD}$ and on sleep disorders are both extensive, relatively little work has explored the intersection of these patient populations, particularly regarding research on patterns of healthcare utilization and expenditures due to insomnia within the $\mathrm{AD}$ population. This research helps to fundamentally characterize this intersecting patient population, which may face unique clinical challenges and benefit from specific treatment strategies. They may also be a chronically underserved group, as insomnia is commonly underdiagnosed and undertreated among elderly populations [19].

In this analysis of the MarketScan databases, we found that $14.6 \%$ of patients newly diagnosed with $\mathrm{AD}$ had a diagnosis of insomnia or received a prescription treatment for insomnia in the 3 months preceding or 12 months following their AD diagnosis. This is consistent with prior claims analyses, which 
Table 2

All-cause healthcare utilization in the 12-month follow-up period

\begin{tabular}{|c|c|c|c|c|c|}
\hline & \multicolumn{2}{|c|}{ Insomnia patients } & \multicolumn{2}{|c|}{ Non-insomnia patients } & \multirow[t]{2}{*}{$p$} \\
\hline & & & & & \\
\hline \multicolumn{6}{|l|}{ Inpatient } \\
\hline Patients with $\geq 1$ inpatient admission $(N, \%)$ & 1,394 & $39.8 \%$ & 3,194 & $32.3 \%$ & $<0.001$ \\
\hline Number of inpatient admissions ${ }^{1}$ (Mean, SD) & 0.6 & 0.9 & 0.4 & 0.7 & $<0.001$ \\
\hline Total days in inpatient setting (Mean, SD) & 9.1 & 13.5 & 7.6 & 11.0 & $<0.001$ \\
\hline \multicolumn{6}{|l|}{ Outpatient (OP) } \\
\hline \multicolumn{6}{|l|}{ Emergency department (ED) visits } \\
\hline Patients with $\geq 1$ ED visit $(N, \%)$ & 1,974 & $56.4 \%$ & 4,744 & $48.0 \%$ & $<0.001$ \\
\hline Number of ED visits ${ }^{1}$ (Mean, SD) & 1.5 & 2.3 & 1.0 & 1.6 & $<0.001$ \\
\hline \multicolumn{6}{|l|}{ OP office visits } \\
\hline Patients with $\geq 1$ office visit $(N, \%)$ & 2,888 & $82.5 \%$ & 8,200 & $83.0 \%$ & 0.546 \\
\hline Number of office visits ${ }^{1}$ (Mean, SD) & 8.8 & 9.1 & 7.5 & 7.6 & $<0.001$ \\
\hline \multicolumn{6}{|l|}{ Skilled nursing facility (SNF) services } \\
\hline Patients with $\geq 1$ SNF claim $(N, \%)$ & 1,490 & $42.6 \%$ & 3,154 & $31.9 \%$ & $<0.001$ \\
\hline Number of SNF claims1 (Mean, SD) & 17.0 & 49.5 & 9.7 & 33.6 & $<0.001$ \\
\hline \multicolumn{6}{|l|}{ Hospice services } \\
\hline Patients with a hospice claim $(N, \%)$ & 29 & $0.8 \%$ & 61 & $0.6 \%$ & 0.188 \\
\hline Number of hospice claims ${ }^{1}$ (Mean, SD) & 0.3 & 6.6 & 0.1 & 2.7 & 0.012 \\
\hline \multicolumn{6}{|l|}{ Other OP services } \\
\hline Patients with $\geq 1$ other OP service $(N, \%)$ & 3,448 & $98.5 \%$ & 9,585 & $97.0 \%$ & $<0.001$ \\
\hline Number of other OP services ${ }^{1}$ (Mean, SD) & 62.1 & 73.1 & 48.6 & 64.6 & $<0.001$ \\
\hline \multicolumn{6}{|l|}{ OP pharmacy } \\
\hline Patients with $\geq 1$ OP claim prescription $(N, \%)$ & 3,409 & $97.4 \%$ & 9,417 & $95.3 \%$ & $<0.001$ \\
\hline Number of claims ${ }^{1}$ (Mean, SD) & 5.3 & 45.2 & 47.8 & 40.5 & $<0.001$ \\
\hline
\end{tabular}

${ }^{1}$ Among all patients.

\begin{tabular}{|ll|}
\hline Inpatient & Outpatient office visit $\quad$ Hospice \\
$\square$ ED visit & SNF \\
$\square$ Outpatient pharmacy & Other outpatient services \\
\hline
\end{tabular}

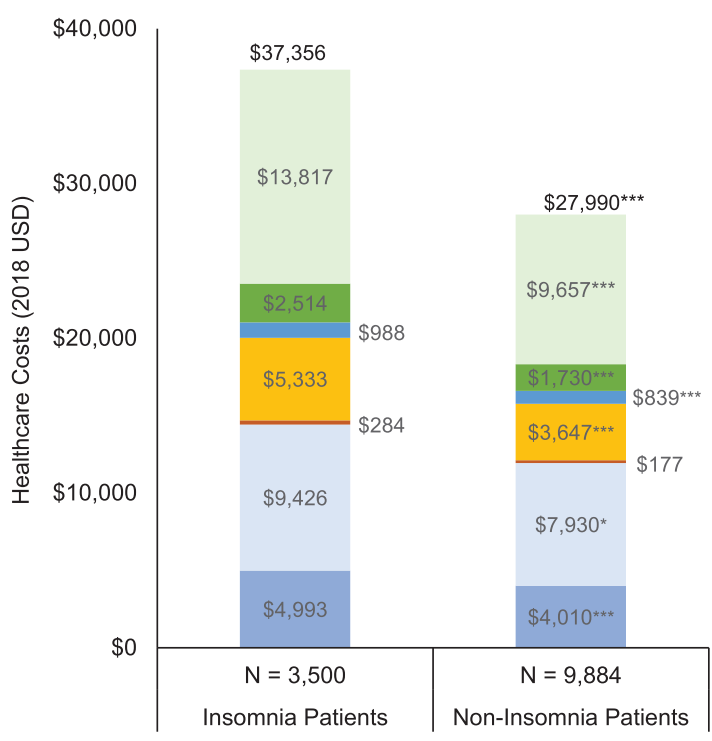

Fig. 2. All-cause healthcare costs per-person per-year in the 12month follow-up period. ED, emergency department; SNF, skilled nursing facility. ${ }^{*} p<0.05,{ }^{* * *} p<0.001$. have reported that $12 \%-13 \%$ of patients with $\mathrm{AD}$ have a comorbid diagnosis of insomnia and $8 \%-17 \%$ have a recent prescription for an insomnia treatment [20-23]. Higher estimates of the prevalence of disturbed sleep among patients with AD have been found through caregiver interviews and other informantbased tools [9-11].

Patients included in this study were on average 82 years old, which is similar to other claims-based studies of newly-diagnosed AD [20-22]. However, a higher percentage were female $(67 \%$ versus $53 \%-63 \%$ ) [21-24], which may reflect the higher prevalence of insomnia among women, as found in other studies $[8,25]$. In addition to being more common, some research suggests that in women insomnia presents in more complex ways with more symptoms [26] and that the adverse effects of insomnia and sleep loss are more severe among women compared to men with regards to cardiovascular health [27, 28], risk of diabetes [28], and psychological affect, functioning, and quality of life [28, 29].

Consistent with prior studies of comorbidity burden among adults with insomnia $[8,15]$, we found that AD patients with comorbid insomnia had higher rates of many comorbidities, including hypertension, hyperlipidemia, and depression, than $\mathrm{AD}$ patients 
Table 3

All-cause healthcare costs in the 12-month follow-up period

\begin{tabular}{|c|c|c|c|c|c|}
\hline \multirow[b]{3}{*}{ Total costs (Mean, SD) } & \multicolumn{2}{|c|}{ Insomnia patients } & \multicolumn{2}{|c|}{ Non-insomnia patients } & \multirow[t]{2}{*}{$p$} \\
\hline & \multicolumn{2}{|c|}{$N=3,500$} & \multicolumn{2}{|c|}{$N=9,884$} & \\
\hline & $\$ 37,356$ & $\$ 70,089$ & $\$ 27,990$ & $\$ 57,179$ & $<0.001$ \\
\hline Median & $\$ 18,813$ & & $\$ 12,318$ & & \\
\hline Inpatient costs (Mean, SD) & $\$ 13,817$ & $\$ 48,854$ & $\$ 9,657$ & $\$ 32,562$ & $<0.001$ \\
\hline Median & $\$ 0$ & & $\$ 0$ & & \\
\hline Outpatient costs (Mean, SD) & $\$ 18,546$ & $\$ 36,351$ & $\$ 14,323$ & $\$ 39,928$ & $<0.001$ \\
\hline Median & $\$ 8,633$ & & $\$ 5,432$ & & \\
\hline ED visit costs (Mean, SD) & $\$ 2,514$ & $\$ 8,932$ & $\$ 1,730$ & $\$ 6,334$ & $<0.001$ \\
\hline Median & $\$ 192$ & & $\$ 0$ & & \\
\hline Outpatient office visit costs (Mean, SD) & $\$ 988$ & $\$ 1,151$ & $\$ 839$ & $\$ 1,007$ & $<0.001$ \\
\hline Median & $\$ 666$ & & $\$ 588$ & & \\
\hline SNF costs (Mean, SD) & $\$ 5,333$ & $\$ 12,988$ & $\$ 3,647$ & $\$ 11,089$ & $<0.001$ \\
\hline Median & $\$ 0$ & & $\$ 0$ & & \\
\hline Hospice costs (Mean, SD) & $\$ 284$ & $\$ 4,121$ & $\$ 177$ & $\$ 3,144$ & 0.112 \\
\hline Median & $\$ 0$ & & $\$ 0$ & & \\
\hline Other outpatient services costs (Mean, SD) & $\$ 9,426$ & $\$ 30,733$ & $\$ 7,930$ & $\$ 36,565$ & 0.030 \\
\hline Median & $\$ 3,873$ & & $\$ 2,789$ & & \\
\hline Outpatient pharmacy costs (Mean, SD) & $\$ 4,993$ & $\$ 8,220$ & $\$ 4,010$ & $\$ 6,876$ & $<0.001$ \\
\hline Median & $\$ 2,981$ & & $\$ 2,216$ & & \\
\hline
\end{tabular}

ED, emergency department; SNF, skilled nursing facility.

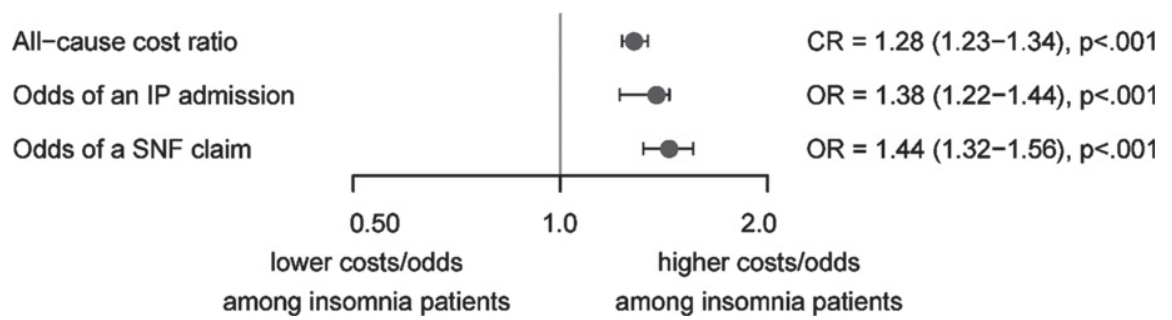

Fig. 3. Multivariable models of total healthcare costs, odds of inpatient (IP) admission, and odds of having a claim for skilled nursing facility (SNF) services with presence of insomnia diagnosis as the primary explanatory variable. CR, cost ratio; OR, odds ratio.

without insomnia. Overall, the demographic and clinical characteristics of the identified population of patients with $\mathrm{AD}$ and comorbid insomnia was consistent with the existing literature on patients with $\mathrm{AD}$ and older adults with insomnia. However, it is important to note that, decreased cerebrovascular health, indicated by diagnoses of hypertension and hyperlipidemia, is itself a risk factor for developing AD [3], possibly compounding the risk of onset or degree of progression of $\mathrm{AD}$ among the insomnia patients.

Among older adults, insomnia severity is positively associated with anxiety, depression, and with the self-reported pain intensity and pain spreading [30]. As comorbidities, depression and insomnia overlap: about $20 \%$ of patients with insomnia also have depression [31]. For those with depression, insomnia can affect the severity of depression symptoms and the course of their mental illness [32]. For those without depression, insomnia is a risk factor of developing depression [33].
In this study, AD patients with insomnia had increased healthcare utilization compared to those without insomnia, including increased utilization of high-cost healthcare services, such as inpatient admissions, emergency room visits, and skilled nursing facility services. This included $32.8 \%$ higher adjusted odds of an inpatient admission and a $43.8 \%$ higher odds of a skilled nursing facility services claim. Prior studies of patients with $\mathrm{AD}$ have reported that $28 \%-40 \%$ of patients newly diagnosed with $\mathrm{AD}$ have an inpatient admission in the first year and $40 \%-47 \%$ have at least one emergency department visit $[7,22]$; in our study the rates were comparable in magnitude with the insomnia sub-group being near the top end or slightly above those ranges. In addition, the difference in utilization between $\mathrm{AD}$ patients with and without insomnia was consistent with a prior study on insomnia in Medicare beneficiaries by Wickwire et al. [8]. They reported that, after adjusting for age, sex, and comorbidities (including 
$\mathrm{AD}$ and related dementias), Medicare beneficiaries with insomnia had $61 \%$ higher odds of an inpatient admission and $49 \%$ higher odds of an ER visit than Medicare beneficiaries without insomnia [8]. Thus, the current work, combined with previous findings, indicates that the already extensive healthcare burden associated with $\mathrm{AD}$ patients can potentially be dramatically increased by comorbid insomnia through the increased need for high-cost services.

We found that unadjusted per-person per-year healthcare costs were $\$ 37,356$ for patients with insomnia but only $\$ 27,990$ for patients without insomnia. After matching for demographics and adjusting for, and comorbidities, this study found that the 1-year incremental cost of insomnia among patients with $\mathrm{AD}$ was $\$ 10,039$. We believe this may be the first such estimate in the research literature, as we could not identify any prior studies examining the incremental burden of insomnia among patients with AD. Independently, both $\mathrm{AD}$ and insomnia are associated with increased costs among older patients [8, 22, 34-37]. Prior estimates of the unadjusted 1-year direct healthcare costs of incident $\mathrm{AD}$ and related dementias have ranged from $\$ 17,712$ to $\$ 48,985$, after adjusting to 2018 USD [22, 35, 38, 39]. Estimates of the adjusted incremental cost of insomnia among older adults vary widely from $\$ 1,143$ among a Medicaid advantage population to $\$ 63,607$ among a random $5 \%$ sample of Medicare [8, 36].

Insomnia in $\mathrm{AD}$ patients also increases the mental and physical burden on home caregivers and reduces their overall quality of life [40-43]. This contributes to the accelerated pathway to high-cost institutionalized care observed among patients with $\mathrm{AD}$ and comorbid insomnia [16]. It is an important topic for future research whether treatment of insomnia can reduce the rate of cognitive decline and delaying the progression of the disease can be important in healthcare cost reduction $[44,45]$. We hypothesize that effective management of insomnia in patients with AD could decrease healthcare costs by reducing the use of high-cost services such as inpatient admissions and skilled nursing facility services and reduce the burden and sleep disturbances among home caregivers. Additional research is needed to examine the relationship between effective insomnia therapy and subsequent healthcare costs.

\section{Limitations}

The limitations of this study include those inherent in any retrospective, claims-based analysis. The
MarketScan Research Databases depend on administrative claims data, and these records are subject to data coding limitations and data entry errors. Cohort misclassification based on off-label insomnia medication utilization or misdiagnosis of insomnia may have occurred. Although we used multivariable analysis to control for differences in baseline characteristics, there are several known factors, including $\mathrm{AD}$ severity and insomnia severity, which may influence outcomes but are not captured in administrative claims and, therefore, could not be controlled for.

In addition, non-prescription medications, such as melatonin, are not captured in claims data. As a result, we may be underestimating costs and misclassifying patients as non-insomnia patients because we are not capturing the use of melatonin as a sleep aid. Furthermore, some insomnia patients are prescribed non-hypnotic drugs (e.g., antihistamines) to improve sleep; these drugs are present in claims data but are less reliably associated with insomnia and so were not used in our patient selection process. Conversely, while the set of drugs that were used as an inclusion criterion for the insomnia cohort are all solely indicated for treating insomnia, off-label use of these medications may have resulted in the misclassification of some patients as having insomnia. As $\mathrm{AD}$ is often diagnosed during a hospitalization for another serious condition, all-cause costs may be overestimated for patients whose insomnia diagnosis occurred on or near their initial AD diagnosis [46]. While this may inflate the overall costs estimated in this study, both cohorts should be impacted similarly due to the patient matching and the method of assigning a proxy insomnia diagnosis date for the control cohort.

Finally, as this study was limited only to those individuals with commercial health coverage or private Medicare supplemental coverage. The results of this analysis may not be generalizable to AD or insomnia patients with other insurance or without health insurance coverage.

\section{CONCLUSIONS}

Comorbid insomnia is associated with a significant healthcare burden among patients with AD. In this claims-based study, patients with $\mathrm{AD}$ and comorbid insomnia had greater utilization of highcost services such as inpatient admissions and skilled nursing facility services than patients without comorbid insomnia. This corresponded with higher total 
healthcare costs among AD patients with comorbid insomnia. These was true across a wide range of medical setting categories including inpatient admissions, emergency room visits, outpatient office visits, and costs associated with skilled nursing facility care. Prior research has examined AD and insomnia separately, but this research helps to characterize the population of $\mathrm{AD}$ patients with comorbid insomnia. The burden of $\mathrm{AD}$ alone is substantial. These findings suggest that comorbid insomnia only creates additional burden to the healthcare system, in addition to increasing caregiver burdens at home and increasing need for institutionalization. There is clear unmet need for properly managing insomnia in patients with underlying $\mathrm{AD}$ or dementia. Slowing the progression of the underlying neurological factors of dementia and treating symptoms that cause additional issues, such as insomnia, should be the focus of new treatment options. Understanding this population, including the interactions of insomnia with $\mathrm{AD}$ and associated treatment needs, has potential to improve the progression of the disease and delay or reduce the need for institutional care.

\section{ACKNOWLEDGMENTS}

This study was funded by Merck Sharp \& Dohme Corp., a subsidiary of Merck \& Co., Inc., Kenilworth, NJ, USA.

Medical writing services were provided Jessamine Winer-Jones of IBM Watson Health. Programming services were provided Paula Smith of IBM Watson Health. Statistical analysis services were provided David Smith of IBM Watson Health. These services were paid for by Merck Sharp \& Dohme Corp., a subsidiary of Merck \& Co., Inc., Kenilworth, NJ, USA.

This research was presented in part at the 2021 Annual Meeting of the American Psychiatric Association conference (online).

Authors' disclosures available online (https:// www.j-alz.com/manuscript-disclosures/21-0713r1).

\section{DATA AVAILABILITY STATEMENT}

The data that support the findings of this study are available from IBM Watson Health. Restrictions apply to the availability of these data, which were used under license for this study.

\section{SUPPLEMENTARY MATERIAL}

The supplementary material is available in the electronic version of this article: https://dx.doi.org/ 10.3233/JAD-210713.

\section{REFERENCES}

[1] Gooch CL, Pracht E, Borenstein AR (2017) The burden of neurological disease in the United States: A summary report and call to action. Ann Neurol 81, 479-484.

[2] Goodman RA, Lochner KA, Thambisetty M, Wingo TS, Posner SF, Ling SM (2017) Prevalence of dementia subtypes in United States Medicare fee-for-service beneficiaries, 2011-2013. Alzheimers Dement 13, 28-37.

[3] Alzheimer's Association (2019) 2019 Alzheimer's disease facts and figures. Alzheimers Dement 15, 321-387.

[4] Hebert LE, Weuve J, Scherr PA, Evans DA (2013) Alzheimer disease in the United States (2010-2050) estimated using the 2010 census. Neurology 80, 1778-1783.

[5] Matthews KA, Xu W, Gaglioti AH, Holt JB, Croft JB, Mack D, McGuire LC (2019) Racial and ethnic estimates of Alzheimer's disease and related dementias in the United States $(2015-2060)$ in adults aged $\geq 65$ years. Alzheimers Dement 15, 17-24.

[6] McKhann GM, Knopman DS, Chertkow H, Hyman BT, Jack CR Jr, Kawas CH, Klunk WE, Koroshetz WJ, Manly JJ, Mayeux R, Mohs RC, Morris JC, Rossor MN, Scheltens P, Carrillo MC, Thies B, Weintraub S, Phelps $\mathrm{CH}$ (2011) The diagnosis of dementia due to Alzheimer's disease: Recommendations from the National Institute on Aging-Alzheimer's Association workgroups on diagnostic guidelines for Alzheimer's disease. Alzheimers Dement 7, 263-269.

[7] Leibson CL, Long KH, Ransom JE, Roberts RO, Hass SL, Duhig AM, Smith CY, Emerson JA, Pankratz VS, Petersen RC (2015) Direct medical costs and source of cost differences across the spectrum of cognitive decline: A population-based study. Alzheimers Dement 11, 917-932.

[8] Wickwire EM, Tom SE, Scharf SM, Vadlamani A, Bulatao IG, Albrecht JS (2019) Untreated insomnia increases allcause health care utilization and costs among Medicare beneficiaries. Sleep 42, zsz007.

[9] Moran M, Lynch CA, Walsh C, Coen R, Coakley D, Lawlor BA (2005) Sleep disturbance in mild to moderate Alzheimer's disease. Sleep Med 6, 347-352.

[10] Carpenter BD, Strauss M, Patterson MB (1996) Sleep disturbances in community-dwelling patients with Alzheimer's disease. Clin Gerontol 16, 35-49.

[11] Webster L, Costafreda Gonzalez S, Stringer A, Lineham A, Budgett J, Kyle S, Barber J, Livingston G (2020) Measuring the prevalence of sleep disturbances in people with dementia living in care homes: A systematic review and meta-analysis. Sleep $\mathbf{4 3}$, zsz251.

[12] Ju YE, Lucey BP, Holtzman DM (2014) Sleep and Alzheimer disease pathology-a bidirectional relationship. Nat Rev Neurol 10, 115-119.

[13] Lim ASP, Kowgier M, Yu L, Buchman AS, Bennett DA (2013) Sleep fragmentation and the risk of incident Alzheimer's disease and cognitive decline in older persons. Sleep 36, 1027-1032. 
[14] Albrecht JS, Wickwire EM, Vadlamani A, Scharf SM, Tom SE (2019) Trends in insomnia diagnosis and treatment among Medicare beneficiaries, 2006-2013. Am J Geriatr Psychiatry 27, 301-309.

[15] Sarsour K, Morin CM, Foley K, Kalsekar A, Walsh JK (2010) Association of insomnia severity and comorbid medical and psychiatric disorders in a health plan-based sample: Insomnia severity and comorbidities. Sleep Med 11, 69-74.

[16] Bianchetti A, Scuratti A, Zanetti O, Binetti G, Frisoni GB, Magni E, Trabucchi M (1995) Predictors of mortality and institutionalization in Alzheimer disease patients 1 year after discharge from an Alzheimer dementia unit. Dementia 6, 108-112.

[17] Shin HY, Han HJ, Shin DJ, Park HM, Lee YB, Park KH (2014) Sleep problems associated with behavioral and psychological symptoms as well as cognitive functions in Alzheimer's disease. J Clin Neurol 10, 203-209.

[18] Consumer Price Index details report tables United States Department of Labor, https://www.bls.gov/cpi/home.htm, Accessed 1 October 2020.

[19] Subramanian S, Surani S (2007) Sleep disorders in the elderly. Geriatrics 62, 10-32.

[20] Bent-Ennakhil N, Coste F, Xie L, Aigbogun MS, Wang Y, Kariburyo F, Hartry A, Baser O, Neumann P, Fillit $\mathrm{H}$ (2017) A real-world analysis of treatment patterns for cholinesterase inhibitors and memantine among newlydiagnosed Alzheimer's disease patients. Neurol Ther 6, 131-144.

[21] Nair R, Haynes VS, Siadaty M, Patel NC, Fleisher AS, Van Amerongen D, Witte MM, Downing AM, Fernandez LAH, Saundankar V, Ball DE (2018) Retrospective assessment of patient characteristics and healthcare costs prior to a diagnosis of Alzheimer's disease in an administrative claims database. BMC Geriatr 18, 243.

[22] Suehs BT, Davis CD, Alvir J, van Amerongen D, Pharmd NC, Joshi AV, Faison WE, Shah SN (2013) The clinical and economic burden of newly diagnosed Alzheimer's disease in a Medicare Advantage population. Am J Alzheimers Dis Other Demen 28, 384-392.

[23] Davis M, O Connell T, Johnson S, Cline S, Merikle E, Martenyi F, Simpson K (2018) Estimating Alzheimer's disease progression rates from normal cognition through mild cognitive impairment and stages of dementia. Curr Alzheimer Res 15, 777-788.

[24] Robinson RL, Rentz DM, Andrews JS, Zagar A, Kim Y, Bruemmer V, Schwartz RL, Ye W, Fillit HM (2020) Costs of early stage Alzheimer's disease in the United States: Crosssectional analysis of a prospective cohort study (GERASUS). J Alzheimers Dis 75, 437-450.

[25] Wickwire EM, Vadlamani A, Tom SE, Johnson AM, Scharf SM, Albrecht JS (2020) Economic aspects of insomnia medication treatment among Medicare beneficiaries. Sleep 43, zsz192.

[26] Jausssent I, Dauvilliers Y, Ancelin ML, Dartigues J-F, Tavernier B, Touchon J, Ritchie K, Besset A (2011) Insomnia symptoms in older adults: Associated factors and gender differences. Am J Geriatr Psychiatry 19, 88-97.

[27] Prather AA, Epel ES, Cohen BE, Neylan TC, Whooley MA (2013) Gender differences in the prospective associations of self-reported sleep quality with biomarkers of systemic inflammation and coagulation: Findings from the Heart and Soul Study. J Psychiatr Res 47, 1228-1235.

[28] Suarez EC (2008) Self-reported symptoms of sleep disturbance and inflammation, coagulation, insulin resistance and psychosocial distress: Evidence for gender disparity. Brain Behav Immun 22, 960-968.

[29] Boccabella A, Malouf J (20174) How do sleep-related health problems affect functional status according to sex? J Clin Sleep Med 13, 685-692.

[30] Dragioti E, Levin LÅ, Bernfort L, Larsson B, Gerdle B (2017) Insomnia severity and its relationship with demographics, pain features, anxiety, and depression in older adults with and without pain: Cross-sectional populationbased results from the PainS65+cohort. Ann Gen Psychiatry 16, 15.

[31] Staner L (2010) Comorbidity of insomnia and depression. Sleep Med Rev 14, 35-46.

[32] Geoffroy PA, Hoertel N, Etain B, Bellivier F, Delorme R, Limosin F, Peyre H (2018) Insomnia and hypersomnia in major depressive episode: Prevalence, sociodemographic characteristics and psychiatric comorbidity in a populationbased study. J Affect Disord 226, 132-141.

[33] Baglioni C, Battagliese G, Feige B, Spiegelhalder K, Nissen C, Voderholzer U, Lombardo C, Riemann D (2011) Insomnia as a predictor of depression: A meta-analytic evaluation of longitudinal epidemiological studies. J Affect Disord 135, 10-19.

[34] Hurd MD, Martorell P, Delavande A, Mullen KJ, Langa KM (2013) Monetary costs of dementia in the United States. New Engl J Med 368, 1326-1334.

[35] Lin PJ, Zhong Y, Fillit HM, Chen E, Neumann PJ (2016) Medicare expenditures of individuals with Alzheimer's disease and related dementias or mild cognitive impairment before and after diagnosis. J Am Geriatr Soc 64, 1549-1557.

[36] Ozminkowski RJ, Wang S, Walsh JK (2007) The direct and indirect costs of untreated insomnia in adults in the United States. Sleep 30, 263-273.

[37] Rattinger GB, Schwartz S, Mullins CD, Corcoran C, Zuckerman IH, Sanders C, Norton MC, Fauth EB, Leoutsakos JM, Lyketsos CG, Tschanz JT (2015) Dementia severity and the longitudinal costs of informal care in the Cache County population. Alzheimers Dement 11, 946-954.

[38] Fishman P, Coe NB, White L, Crane PK, Park S, Ingraham B, Larson EB (2019) Cost of dementia in Medicare managed care: A systematic literature review. Am J Manag Care 25, e247-e253.

[39] Joyce AT, Zhao Y, Bowman L, Flynn JA, Carter CT, Ollendorf DA (2007) Burden of illness among commercially insured patients with Alzheimer's disease. Alzheimers Dement 3, 204-210.

[40] Okuda S, Tetsuka J, Takahashi K, Toda Y, Kubo T, Tokita S (2019) Association between sleep disturbance in Alzheimer's disease patients and burden on and health status of their caregivers. J Neurol 266, 1490-1500.

[41] McCurry SM, Logsdon RG, Teri L, Gibbons LE, Kukull WA, Bowed JD, McCormick WC, Larson EB (1999) Characteristics of sleep disturbance in community-dwelling Alzheimer's disease patients. J Geriatr Psychiatry Neurol 12, 53-59.

[42] Creese J, Bédard M, Brazil K, Chambers L (2008) Sleep disturbances in spousal caregivers of individuals with Alzheimer's disease. Int Psychogeriatr 20, 149-161.

[43] Gehrmann P, Gooneratne NS, Brewster GS, Richards KC, Karlawish J (2018) Impact of Alzheimer disease patients' sleep disturbances on their caregivers. Geriatr Nurs 39, 60-65.

[44] Lenox-Smith A, Reed C, Lebrec J, Belger M, Jones RW (2018) Potential cost savings to be made by slowing cognitive decline in mild Alzheimer's disease dementia using 
a model derived from the UK GERAS observational study. BMC Geriatrics 18, 57.

[45] Stefanacci RG (2011) The costs of Alzheimer's disease and the value of effective therapies. Am J Manag Care 17 ( Suppl 13), S356-S362.
[46] Deb A, Thornton JD, Sambamoorthi U, Innes K (2017) Direct and indirect cost of managing Alzheimer's disease and related dementias in the United States. Expert Rev Pharmacoecon Outcomes Res 17, 189-202. 